\title{
Explosive Electric Breakdown due to Conducting-Particle Deposition on an Insulating Substrate
}

\author{
Cláudio L. N. Oliveira, ${ }^{1, *}$ Nuno A. M. Araújo, ${ }^{2,3, \dagger}$ José S. Andrade, Jr., ${ }^{1,2, \$}$ and Hans J. Herrmann ${ }^{2,1, \S}$ \\ ${ }^{1}$ Departamento de Física, Universidade Federal do Ceará, 60451-970 Fortaleza, Ceará, Brazil \\ ${ }^{2}$ Computational Physics, IfB, ETH Zürich, Hönggerberg, CH-8093 Zürich, Switzerland \\ ${ }^{3}$ Departamento de Física, Faculdade de Ciências, Universidade de Lisboa, P-1749-016 Lisboa, Portugal \\ and Centro de Física Teórica e Computacional, Universidade de Lisboa, \\ Avenida Professor Gama Pinto 2, P-1649-003 Lisboa, Portugal
}

(Received 10 July 2014; published 10 October 2014)

\begin{abstract}
We introduce a theoretical model to investigate the electric breakdown of a substrate on which highly conducting particles are adsorbed and desorbed with a probability that depends on the local electric field. We find that, by tuning the relative strength $q$ of this dependence, the breakdown can change from continuous to explosive. Precisely, in the limit in which the adsorption probability is the same for any finite voltage drop, we can map our model exactly onto the $q$-state Potts model and thus the transition to a jump occurs at $q=4$. In another limit, where the adsorption probability becomes independent of the local field strength, the traditional bond percolation model is recovered. Our model is thus an example of a possible experimental realization exhibiting a truly discontinuous percolation transition.
\end{abstract}

DOI: 10.1103/PhysRevLett.113.155701

PACS numbers: 64.60.ah, 05.50.+q, 89.75.Da

One of the main problems in the manufacturing of integrated circuits (IC), where millions of nanometric metallic and semiconductor devices are placed on a substrate, is pollution with metallic dust, since it can induce an electric breakdown, leading to malfunctioning and a shorter lifetime of the IC [1-3]. The following question then arises: under which conditions and how fast does such a system collapse. To answer this question, one needs to take into account that the deposition of metallic particles is hindered by the local electric field. This strongly nonlinear interplay between adsorption and the local geometry gives rise to interesting phenomena that we will explore here with the help of a rather simple model, which, however, can still capture the essential physics of the problem. In particular, we find that the collapse can either be continuous or explosive depending on the physical parameters.

Here, we model the substrate as an $L \times L$ tilted square lattice with periodic boundary conditions in one direction and a voltage drop $V_{0}$ applied in the other direction. For simplicity, the bonds of the lattice can be either associated with highly resistive elements or metallic particles, with resistances $R=1$ and 0 , respectively, and $V_{0}$ is set to unity. Nodes connected by metallic bonds constitute metallic clusters and thus have the same electric potential. Resistive bonds that connect sites of the same metallic cluster are called internal bonds, while all other resistive bonds are called merging bonds. Internal bonds do not feel any field and therefore metal dust can be adsorbed on them with a probability $q$ times higher than on merging bonds, where the factor $q>1$ describes the relative deposition disadvantage due to the presence of the field. Additionally, the probability of adsorbing a metallic particle decreases monotonically with the strength of the local field gradient $\Delta V$; namely, we will assume here generically that the adsorption probability decays as $1-\left(\Delta V / V_{0}\right)^{\gamma}$, where $\gamma$ is another adjustable parameter. In the framework of this model, it follows that the probability for metallic bonds to replace resistive bonds (adsorption process) is given by

$$
W=\frac{p}{q}\left[1+(q-1)\left(1-\left(\frac{\Delta V}{V_{0}}\right)^{\gamma}\right)\right]
$$

where $p$ is the adsorption probability in the absence of voltage drop. Thus if the bond is internal, the adsorption probability becomes $W=p$, since $\Delta V=0$.

The inverse process, namely, desorption then happens naturally with a probability 1 minus the probability of adsorption: $1-p$. The model has three parameters: $p$ is a measure for the amount of metallic dust, $q$ is the enhancement of adsorption if there is no local electric field, and $\gamma$ is the dependence on the strength of the local field. For $\gamma=\infty$, all resistive bonds (merging and internal) can be replaced with the same probability $p$ so that, in this limit, one recovers classical bond percolation [4]. For $q=1$, classical bond percolation is also recovered for any value of $\gamma$. Moreover, as we will discuss later, one recovers the $q$-state Potts model for $\gamma=0[5,6]$. In a way, our model is the inverse of a random fuse model [7-10]. In the latter, depending on the local potential drop, bonds can only be removed, while here the local field solely affects the addition of bonds.

The simulations are performed here by randomly choosing, at each iteration, a bond between neighboring nodes $i$ and $j$ and attempting to change its state according to the 


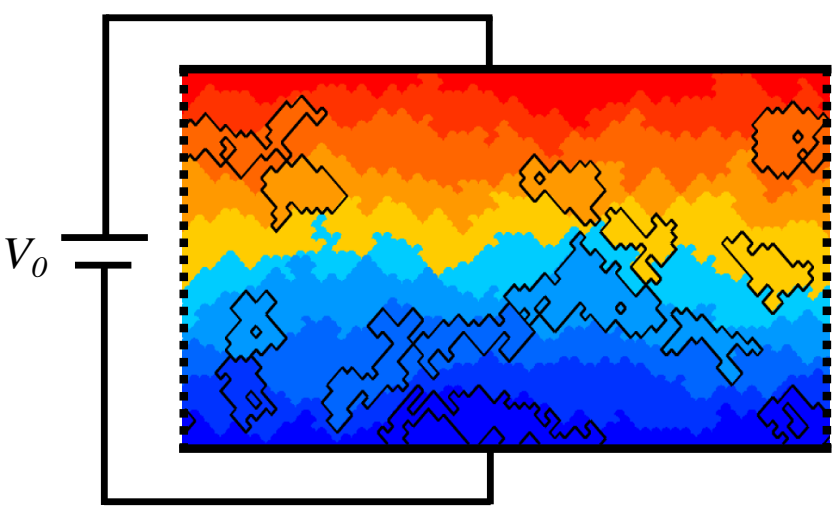

FIG. 1 (color online). Plot showing a typical state of the system for $q=10, L=128, \gamma=0.1$, and $p=0.57$. A potential drop $V_{0}$ is applied from top to bottom and periodic boundary conditions from left to right. The nodes are colored according to their corresponding potential, with the constraint that nodes belonging to the same metallic cluster hold the same potential. Nodes with high potential are shown in red (top), while low potential ones are presented in blue (bottom). The boundaries of several metallic clusters are highlighted for better visualization.

probabilities previously described. Each time a merging bond is identified, the local potential drop $|\Delta V|_{i j}$ is calculated by solving the Kirchhoff equations for each node simultaneously. This is equivalent to solve the Laplace equation $\nabla^{2} V=0$ [11] by discretization, and impose that nodes belonging to the same metallic cluster have the same potential, as illustrated in Fig. 1.

Regardless of the starting configuration, a steady state is reached after a certain number of iterations. In Fig. 2 we show how the fraction $P$ of nodes in the largest metallic cluster fluctuates with the number of iterations $n$, at the steady state, for $q=10$, and different values of $p$ and $\gamma$. For fixed values of $q$ and $\gamma$, by increasing $p$ one reaches a value $p_{c}(q, \gamma)$ at which the system electrically breaks down. At this point, a spanning metallic cluster can appear after steady state, which makes the system fluctuate strongly between resistive and metallic configurations (see Fig. 2). In particular, $p_{c}(q, \gamma=\infty)=1 / 2$ because, as previously stated, for $\gamma=\infty$ our model recovers bond percolation on the square lattice. For $\gamma=0.1$, as shown in Fig. 2(a), $P$ mainly oscillates around two well-defined values, $P \approx 0.05$ and 0.45 , yielding a bimodal distribution [see Fig. 2(c)]. As we show later, the fact that the height of the peaks grows with $L^{d / 2}[12,13]$ and the distance between peaks does not vanish with system size are clear signatures of a discontinuous transition. By contrast, for $\gamma=1$ [see Fig. 2(b)], although the variable $P$ also fluctuates around an average value, the distribution of $P$ is unimodal and there is no sign of metastability [see Fig. 2(d)]. Thus, the transition in this case is continuous. Shown in Fig. 3 are snapshots of steady state configurations for both cases at and around the threshold $p_{c}$. While for $\gamma=0.1$ a compact gigantic metallic cluster abruptly appears suggesting a discontinuous transition [see Figs. 3(a)-3(c)], for $\gamma=1$ the largest cluster looks fractal and grows with $p$ suggesting a continuous transition [see Figs. 3(d)-3(e)]. As a consequence, the electric breakdown is either smooth or explosive depending on the values of $\gamma$ and $q$.

The phase transition between resistive and metallic states is described in terms of the average fraction of nodes in the largest metallic cluster $\langle P\rangle$ taken here as the order parameter. For a given pair of $p$ and $\gamma$ values, we calculate $\langle P\rangle$ by averaging $P$ over many iterations $n$ at the steady state. Notice that the steady state may be reached with more or
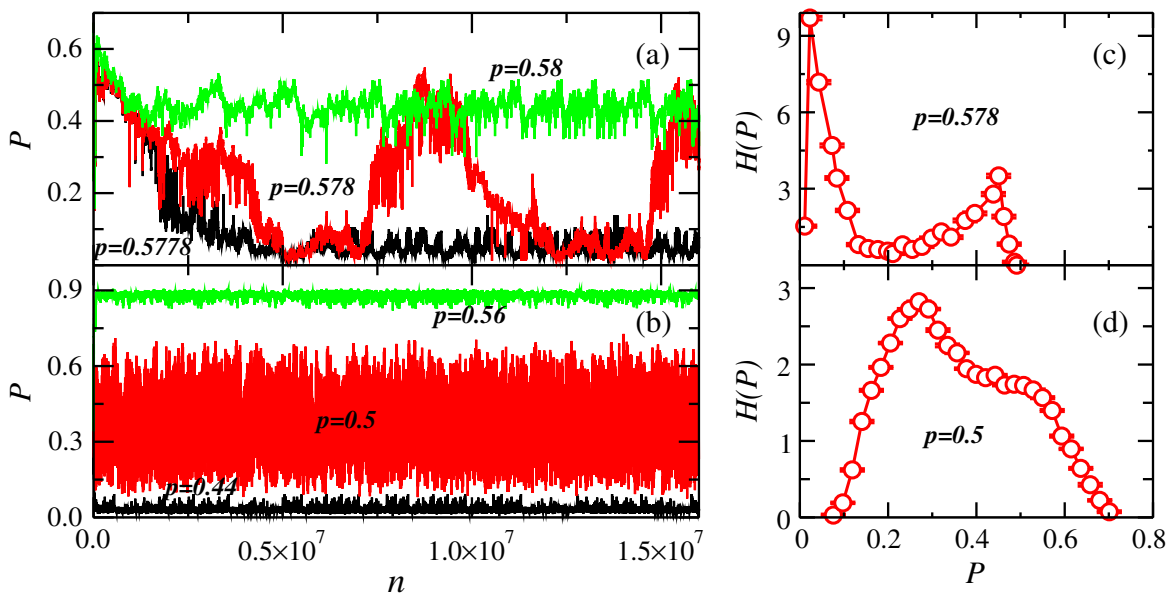

FIG. 2 (color online). (a) Fraction $P$ of nodes in the largest cluster with the number of iterations $n$ (sampled bonds) for $q=10$, $L=128, \gamma=0.1$, and distinct values of $p$ below (black), above (green), and at the transition point $p_{c}=0.578$ (red). (b) The same as in (a) but for $\gamma=1$ and $p_{c}=0.5$. The histograms for $P$ at the transition point are shown in (c) and (d) for $\gamma=0.1$ and $\gamma=1$, respectively. For $\gamma=0.1$, the histogram is bimodal and the evolution is characterized by metastability, two typical signs of a discontinuous transition. By contrast, for $\gamma=1$ the transition is continuous since the histogram is unimodal and there is no evidence of metastability. Error bars show the standard error within each bin of the histogram. 


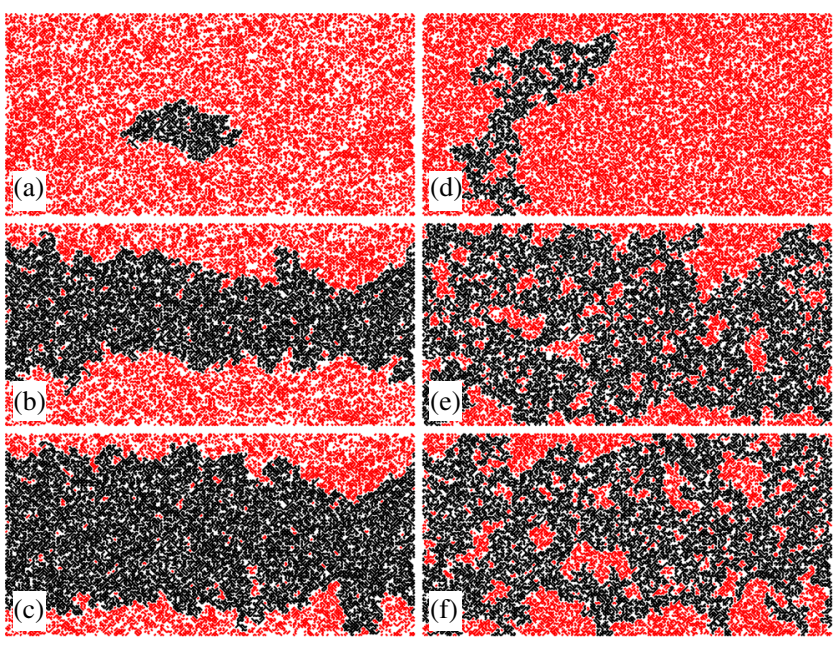

FIG. 3 (color online). Snapshots of steady state configurations for $q=10, L=128, \gamma=0.1$, with (a) $p=0.57$, (b) $p=0.58$, and (c) $p=0.59$, and for $\gamma=1$ with (d) $p=0.49$, (e) $p=0.5$, and (f) $p=0.51$. Metallic bonds belonging to the largest cluster are colored in black, while the bonds belonging to the other metallic cluster appear in red. For $\gamma=0.1$, the largest metallic cluster is compact and grows abruptly at the threshold, $p_{c} \approx 0.58$, while for $\gamma=1$ it is fractal and grows rather smoothly with $p$.

less iterative steps depending on the parameters of the system. In Fig. 2(a), for example, not less than $3 \times 10^{6}$ steps were needed. In addition, well-defined histograms with reduced fluctuations can only be obtained in some cases for $n>10^{7}$ steps. Since most of these steps involve the inversion of large matrices, the calculation becomes prohibitively heavy for system sizes $L>128$. In Fig. 4 we show $\langle P\rangle$ as a function of $p$ for different values of $\gamma$, for $q=2$ in (a) and $q=10$ in (b). As depicted, the value of $p_{c}$ decreases with $\gamma$ and increases with $q$, being always between the critical points of bond percolation, $p_{c}=1 / 2$, and the one of the corresponding $q$-state Potts model (vertical lines in the plots).

In the particular case of $\gamma=0$, merging bonds become metallic with probability $p / q$ and internal bonds with probability $p$. This is precisely the Monte Carlo procedure to obtain the Coniglio-Klein clusters [14] for the $q$-state Potts model as derived by Gliozzi $[6,15]$ from the Kasteleyn-Fortuin formulation [16,17]. Thus, $\langle P\rangle$ is nothing but the order parameter of the $q$-state Potts model for $\gamma=0$. From self-duality, the transition point is known exactly for the square lattice to be [17],

$$
p_{c}(q, \gamma=0)=\frac{\sqrt{q}}{1+\sqrt{q}} .
$$

Furthermore it is known that, in two dimensions, the phase transition of the Potts model changes at $q=4$ from second order, for $q<4$, to first order, for $q>4$. Our physical model then encompasses a plethora of transitions between continuous and abrupt electric breakdowns.

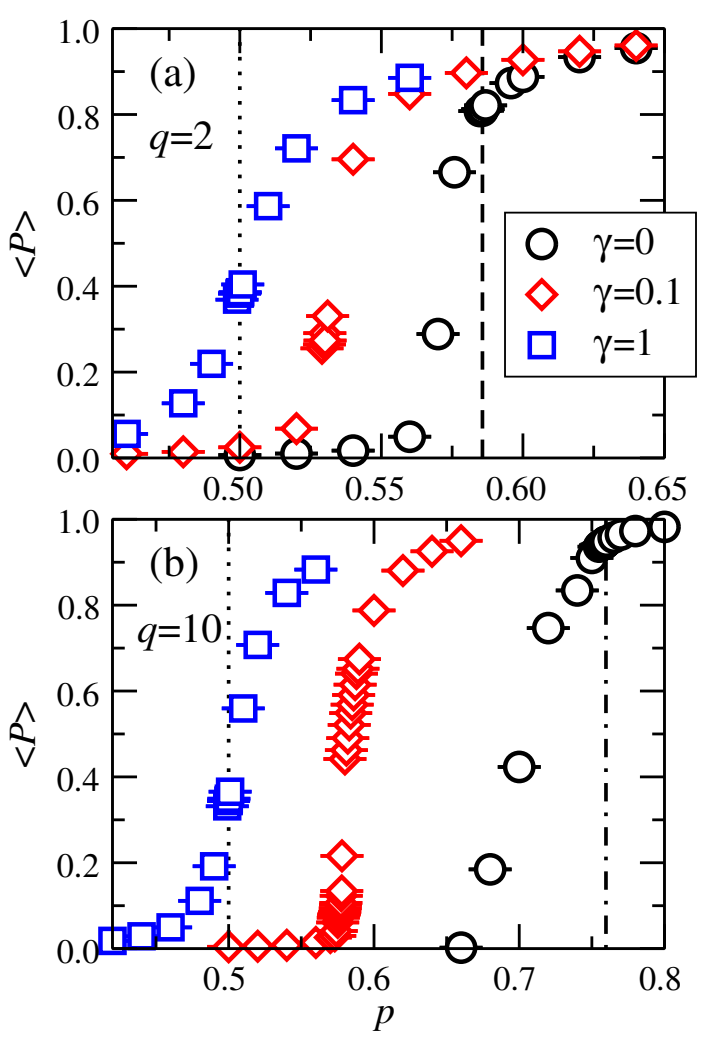

FIG. 4 (color online). Dependence on $p$ the average fraction $\langle P\rangle$ of nodes in the largest cluster for $\gamma=0,0.1$, and 1 , for $q=10$ (a) and $q=2$ (b), with $L=128$. The transition point for a given $\gamma$ and $q$ is bounded between the critical point for bond percolation, $p_{c}(q=1, \gamma=0)=p_{c}(q, \gamma=\infty)=1 / 2$ (dotted lines), and the critical point for the $q$-state Potts model, given by Eq. (2), where $p_{c}(q=2, \gamma=0) \approx 0.5858$ (dashed line), and $p_{c}(q=10$, $\gamma=0) \approx 0.7597$ (dotted-dashed line). Error bars show the standard error for a given value of $p$.

Remarkably, for the case $\gamma=0$, this is not a numerical but an exact result. It constitutes in fact a beautiful example for explosive (discontinuous) percolation $[18,19]$ which has an analytical approach on one hand, and an experimental realization on the other.

We investigate numerically if this transition from continuous to abrupt exists for experimentally relevant values of $\gamma$, namely, $\gamma \neq 0$. The two different patterns for the evolution of $P$ shown in Fig. 2 suggest this behavior; however, a more systematic study based on size scaling is necessary. One should note that, as shown in Fig. 4, due to the finite size of the simulated systems, the numerical data for $\gamma=0$ (circles) still deviate considerably from those expected in the thermodynamic limit, as given by Eq. (2) (marked by dashed lines). In particular, one cannot recognize the predicted continuous (discontinuous) transition in Fig. 4(a) [Fig. 4(b)]. Therefore, we studied the histogram of the order parameter at $p_{c}$ for different system sizes, as shown in Fig. 5 for the case $\gamma=0.1$ and $q=10$. Here, $p_{c}$ was determined from a finite-size extrapolation of the inflection points of $P$. Figure 5 clearly shows a typical 

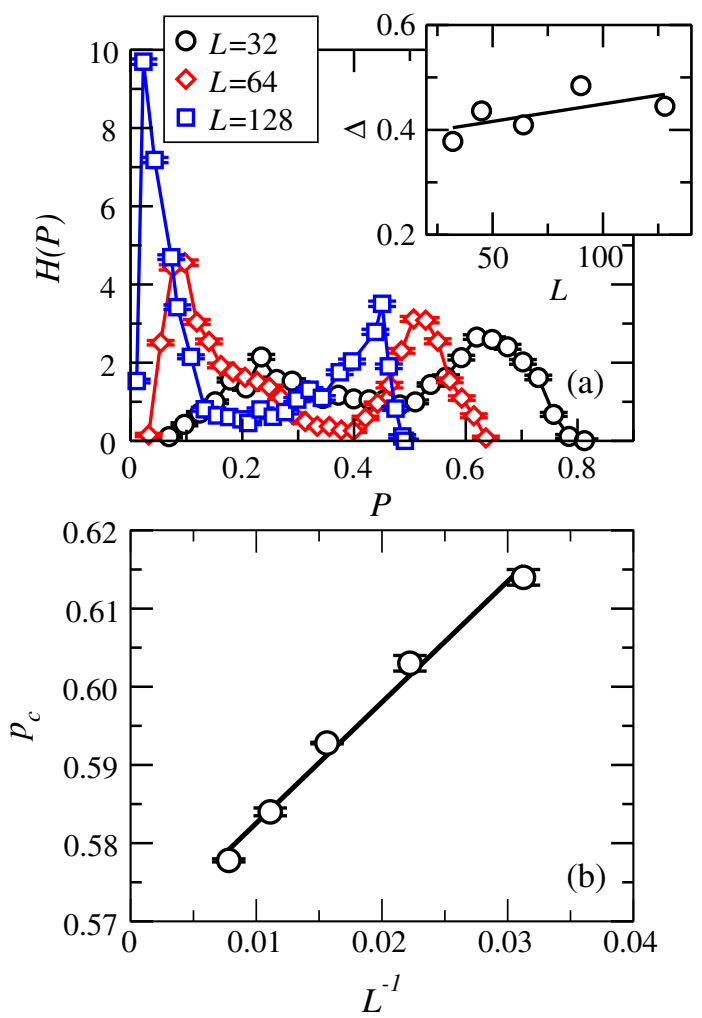

FIG. 5 (color online). (a) Histogram of the fraction $P$ of nodes in the largest metallic cluster for different system sizes $L$ at $p_{c}$ for $q=10$ and $\gamma=0.1$. In the inset of (a), $\Delta$ is the difference between the two peaks in the histogram. One clearly sees that $\Delta$ does not decrease with the system size, as one would expect for a continuous transition. For every $L, p_{c}(L)$ is obtained as the value of $p$ that maximizes the derivative of $P, d P / d p$. As shown in (b), $p_{c}$ can then be extrapolated to the thermodynamic limit through $p_{c}=0.5671+1.5463 L^{-1}$. The error bars shown in (a) are the standard error within each bin of the histogram while in (b) they represent the error of the finite difference approximation to compute the derivative of $p$.

bimodal distribution. Analyzing the trend of the data with system size yields that the distance between peaks does not vanish in the thermodynamic limit while the peaks increase, within the statistical error bars, linearly $\left(\sim L^{d / 2}\right)$ with system size giving very strong support for the presence of a discontinuous transition. Because of the large numerical effort involved in calculating the local voltages [20], we refrained from mapping in detail the full transition surface in the three-dimensional $(p, q, \gamma)$ phase diagram. The important conclusion we extract from Fig. 5 is that also for $\gamma \neq 0$ (in this case $\gamma=0.1$ ) one can find an explosive electric breakdown.

In summary, we have discovered that the electric breakdown due to pollution with metallic powder can become explosive if the inhibition of adsorption due to a local electric field becomes too strong. In the case $\gamma=0$, i.e., when the details of the field strength become unimportant, the model can be solved exactly, representing one of the rare examples where an explosive percolation transition at a finite $p_{c}$ can be proven to exist. The physical reason why the electric breakdown becomes abrupt is the same as for other percolation models [21-23], namely, avoiding the occupation of bonds that locally feel an electric field prevents the early appearance of an infinite cluster. This effect gets more pronounced the more large local fields matter, i.e., the more cutting bonds are particularly punished. In fact, finding possible mechanisms leading to abrupt percolation transitions is the subject of enthusiastic discussions in the recent literature [21-33]. For simplicity, here we consider a general power-law form for the decay of the adsorption probability with the potential drop. However, our results can be straightforwardly extended to any strictly decaying function $f(\Delta V)$, with its values bounded between zero and unity. In the limit $f(\Delta V)=1$, classical percolation is recovered, while $(\Delta V)=0$ corresponds to the $q$-state Potts model.

The finding of the explosive electric breakdown phenomenon may explain the difficulties in predicting the failure of electronic circuits. It might, on the other hand, also help to mitigate the problem by working under conditions corresponding to noncritical regions in phase space. It is therefore interesting to further explore the present model and, in particular, to incorporate into it more empirical information about real IC circuits.

We thank the Brazilian Agencies Conselho Nacional de Desenvolvimento Científico e Tecnológico (CNPq), Coordenação de Aperfeiçoamento de Pessoal de Nível Superior (CAPES), and Fundação Cearense de Apoio ao Desenvolvimento Científico e Tecnológico (FUNCAP), the National Institute of Science and Technology for Complex Systems in Brazil, the European Research Council (ERC) Advanced Grant No. 319968-FlowCCS for financial support, and the Portuguese Foundation for Science and Technology (FCT) under Contracts No. IF/00255/2013, No. PEst-OE/FIS/UI0618/2014, and No. EXCL/FIS-NAN/ 0083/2012.

*lucas@fisica.ufc.br

†nmaraujo@fc.ul.pt

†soares@fisica.ufc.br

§hans@ifb.baug.ethz.ch

[1] M. A. Alam, R. K. Smith, B. E. Weir, and P. J. Silverman, Nature (London) 420, 378 (2002).

[2] L. Niemeyer, L. Pietronero, and H. J. Wiesmann, Phys. Rev. Lett. 52, 1033 (1984).

[3] J. F. Verweij and J. H. Klootwijk, Microelectron. J. 27, 611 (1996).

[4] D. Stauffer and A. Aharony, Introduction to Percolation Theory (Taylor \& Francis, London, 1992).

[5] R. B. Potts, Proc. Cambridge Philos. Soc. 48, 106 (1952).

[6] F. Gliozzi, Phys. Rev. E 66, 016115 (2002).

[7] B. Kahng, G. G. Batrouni, S. Redner, L. de Arcangelis, and H. J. Herrmann, Phys. Rev. B 37, 7625 (1988). 
[8] J. S. Andrade, H. J. Herrmann, A. A. Moreira, and C. L. N. Oliveira, Phys. Rev. E 83, 031133 (2011).

[9] N. Posé, N. A. M. Araújo, and H. J. Herrmann, Phys. Rev. E 86, 051140 (2012).

[10] A. A. Moreira, C. L. N. Oliveira, A. Hansen, N. A. M. Araújo, H. J. Herrmann, and J. S. Andrade, Phys. Rev. Lett. 109, 255701 (2012).

[11] The linear equation system was solved through the HSL library, a collection of FORTRAN codes for large-scale scientific computation. See http://www.hsl.rl.ac.uk/.

[12] K. Binder and D. P. Landau, Phys. Rev. B 30, 1477 (1984).

[13] C. Borgs, R. Kotecký, and S. Miracle-Solé, J. Stat. Phys. 62, 529 (1991).

[14] A. Coniglio and W. Klein, J. Phys. A 13, 2775 (1980).

[15] J.-S. Wang, O. Kozan, and R. H. Swendsen, Phys. Rev. E 66, 057101 (2002).

[16] C. M. Fortuin and P. W. Kasteleyn, Physica (Amsterdam) 57, 536 (1972).

[17] F. Y. Wu, Rev. Mod. Phys. 54, 235 (1982); J. Stat. Phys. 18, 115 (1978).

[18] R. J. Baxter, J. Phys. C 6, L445 (1973).

[19] B. Nienhuis, A. N. Berker, E. K. Riedel, and M. Schick, Phys. Rev. Lett. 43, 737 (1979).

[20] The data shown in Fig. 5 represent several months of calculation. For $L=128$, each sample takes around 20 days in one Intel Xeon $51603.0 \mathrm{GHz}$ processor.
[21] N. A. M. Araújo and H. J. Herrmann, Phys. Rev. Lett. 105, 035701 (2010).

[22] Y.S. Cho, S. Hwang, H. J. Herrmann, and B. Kahng, Science 339, 1185 (2013).

[23] W. Chen and R. M. D’Souza, Phys. Rev. Lett. 106, 115701 (2011).

[24] D. Achlioptas, R. M. D’Souza, and J. Spencer, Science 323, 1453 (2009).

[25] R. M. Ziff, Phys. Rev. Lett. 103, 045701 (2009).

[26] R. M. D’Souza and M. Mitzenmacher, Phys. Rev. Lett. 104, 195702 (2010).

[27] R. A. da Costa, S. N. Dorogovtsev, A. V. Goltsev, and J.F.F. Mendes, Phys. Rev. Lett. 105, 255701 (2010).

[28] O. Riordan and L. Warnke, Science 333, 322 (2011).

[29] J. Nagler, A. Levina, and M. Timme, Nat. Phys. 7, 265 (2011).

[30] A. A. Moreira, E. A. Oliveira, S. D. S. Reis, H. J. Herrmann, and J. S. Andrade Jr., Phys. Rev. E 81, 040101 (2010).

[31] Y. S. Cho, J. S. Kim, J. Park, B. Kahng, and D. Kim, Phys. Rev. Lett. 103, 135702 (2009); Y. S. Cho, B. Kahng, and D. Kim, Phys. Rev. E 81, 030103 (2010).

[32] S. D. S. Reis, A. A. Moreira, and J. S. Andrade, Phys. Rev. E 85, 041112 (2012).

[33] N. A. M. Araújo, J. S. Andrade, R. M. Ziff, and H. J. Herrmann, Phys. Rev. Lett. 106, 095703 (2011). 